\title{
CONTRIBUIÇÕES DA ASSISTÊNCIA FARMACÊUTICA NO ENFRENTAMENTO DA COVID-19 EM SOBRAL, CEARÁ
}

CONTRIBUTIONS OF THE PHARMACEUTICAL ASSISTANCE IN COPING WITH COVID-19 IN SOBRAL, CEARÁ

CONTRIBUCIONES DE ASISTENCIA FARMACÉUTICA PARA ENFRENTAR LA COVID-19 EN SOBRAL, CEARÁ

Estevam Ferreira da Ponte Neto 1

Tereza Doralúcia Rodrigues Ponte ${ }^{2}$

Delano de Sousa Aragão ${ }^{3}$

Pedro Henrique Martins ${ }^{4}$

Palavras-chave:

Assistência farmacêutica; Gestão em saúde; Infecções por coronavírus.

Keywords:

Pharmaceutical assistance; Health management; Coronavirus infections.

Palabras clave: Asistencia farmacéutica; Manejo de la salud; Infecciones por coronavirus;

Submetido: $14 / 11 / 20$

Aprovado: $22 / 12 / 20$

Autor(a) para Correspondência: Estevam Ferreira da Ponte Neto Rua Vereador Raimundo Nilo Donizete, 15, Junco, Sobral-CE CEP: 62.030-495 E-mail: estevam_ponte@hotmail.com

\section{RESUMO}

Este estudo relata as contribuições da Coordenadoria da Assistência Farmacêutica no enfrentamento da Covid-19 no município de Sobral, Ceará. Trata-se de um relato de experiencia compreendendo as ações executadas no período de março a outubro de 2020, sob o olhar de profissionais que vivenciaram o processo junto à rede de serviços de referência para a Covid-19, vinculados à Secretaria Municipal da Saúde. No cenário da pandemia de Covid-19, a assistência farmacêutica despontou com um importante papel, assegurando as ações programadas com vistas à integralidade $e$ universalidade do cuidado e atendendo às necessidades advindas da nova situação epidemiológica, a exemplo da retaguarda aos hospitais de campanha e apoio central nos processos logísticos para toda a rede de serviços de saúde, requerendo o estabelecimento da remodelagem de fluxos e processos de trabalho, considerando sempre o direito universal à saúde, os princípios norteadores do Sistema Único de Saúde e as diretrizes para uma segurança à assistência farmacêutica.

1. Farmacêutico. Especialista em Citologia Oncótica e Oncohematologia. Coordenador da Assistência Farmacêutica de Sobral. Secretaria de Saúde de Sobral. Prefeitura Municipal de Sobral. E-mail: estevam_ponte@hotmail.com ORCID: https://orcid.org/0000-0003-1733-8895

2. Farmacêutica. Mestranda em Gestão em Saúde (UECE). Gerente de Centro de Saúde da Família. Secretaria da Saúde de Sobral. Prefeitura Municipal de Sobral. E-mail: terezadoralucia@hotmail.com. ORCID: https://orcid. org/0000-0003-2979-0766

3. Farmacêutico. Especialista Hematologia Clínica. Gerente de Aquisição da Central de Abastecimento Farmacêutica. Secretaria de Saúde de Sobral, e-mail: delanoaragao@bol.com.br ORCID: https://orcid.org/0000-0002-0005-6122 4. Farmacêutico. Especialista em Auditoria de sistemas e Serviços de Saúde Gerente da Farmácia de Medicamentos Especiais. Secretaria de Saúde de Sobral. Prefeitura Municipal de Sobral, E-mail: phmartins2686@gmail.com.0RCID: https://orcid.org/0000-0002-8038-9073 


\section{ABSTRACT}

This study reports the contributions of the Pharmaceutical Assistance Coordination in facing Covid-19 in the municipality of Sobral, Ceará. This is an experience report comprising the actions carried out from March to 0ctober 2020, under the supervision of professionals who experienced the process with the network of reference services for Covid-19, linked to the Municipal Health Department. In the Covid-19 pandemics scenario, pharmaceutical assistance has emerged with an important role: to ensure the actions programmed aiming the integrality and universality of health care, and to meet the needs arising from the new epidemiological status, such as the support of campaign hospitals and the central support of logistical processes for the entire health service network, requiring the establishment of work flow and process remodeling, always considering the universal right to health, the guiding principles of the Unified Health System and the guidelines for pharmaceutical assistance security.

\section{RESUMEN}

Este estudio relata los aportes de la Coordinación de Asistencia Farmacéutica (Coordenadoria da Assistência Farmacêutica) en el enfrentamiento a la Covid-19 en el municipio de Sobral, Ceará. Se trata de un relato de experiencia que recoge las acciones realizadas desde marzo a octubre de 2020, bajo la mirada de los profesionales que vivieron el proceso con la red de servicios de referencia para la Covid-19, vinculada al Departamento Municipal de Salud. En el escenario de la pandemia de la Covid-19, la asistencia farmacéutica ha emergido con un papel importante, asegurando las acciones planificadas con miras a la integralidad y universalidad de la atención y atendiendo las necesidades derivadas de la nueva situación epidemiológica, como la retaguardia de los hospitales en Campaña y apoyo central en procesos logísticos para toda la red de servicios de salud, requiriendo el establecimiento de la remodelación de flujos y procesos de trabajo, considerando siempre el derecho universal a la salud, los principios rectores del Sistema Único de Saúde (SUS) y lineamientos para la seguridad de la asistencia farmacéutica.

\section{INTRODUÇÃO}

A pandemia por Covid-19, em 2020, impôs desafios à gestão e atenção à saúde. Neste escopo, inserese a assistência farmacêutica. Esta é compreendida como um conjunto de ações voltadas à promoção, proteção e recuperação da saúde, tanto individual como coletiva, tendo o medicamento como insumo essencial e visando ao acesso e ao seu uso racional. Conjunto esse que requer desenvolvimento científico e tecnológico com a produção de medicamentos e insumos, bem como demais ações de seleção, programação, aquisição, distribuição, dispensação, garantia da qualidade de produtos e serviços, acompanhamento e avaliação da sua utilização, na perspectiva da obtenção de resultados concretos e da melhoria da qualidade de vida da população ${ }^{1}$.

Esse escopo de ações integra o cotidiano da Coordenadoria da Assistência Farmacêutica; no entanto, tais ações multiplicaram-se no curso da pandemia, bem como foram agregados novos desafios, espelhando uma situação mundial de emergência em saúde pública. Nesse contexto, a literatura tem destacado: desabastecimento de medicamentos necessários à terapêutica para a Covid-19 e até mesmo daqueles de uso regular; insuficiência de disponibilização de produtos médico-hospitalares, com especial atenção para os equipamentos de proteção individual (EPI), elementos essenciais ao exercício profissional dos trabalhadores; limitada disponibilidade de insumos estratégicos para a garantia da continuidade e integralidade da atenção à saúde de pacientes de uso contínuo de medicamentos; e os recém-designados profiláticos para o tratamento da Covid-19².

Reconhece-se que 0 desabastecimento de medicamentos é um problema mundial recorrente e que se intensifica em situações agudas, não constantes no planejamento em saúde. Na década de 1950 já era possível encontrar relatos sobre a discussão desse problema em âmbito global. Na época, entendido como resultado do súbito aumento da demanda ou pela fragilidade dos sistemas de saúde, resultantes de financiamento insuficiente, bem como de compras inadequadas e/ou sistemas de distribuição ineficientes ${ }^{3}$. Cinquenta anos depois, foram vividas situações similares com análise dos condicionantes ainda não realizada, mas com repercussões diretas na promoção da assistência à saúde. 
Com a intenção de se antever, quando possível, e de responder efetivamente às necessidades por assistência farmacêutica, é que a Coordenadoria da Assistência Especializada visou, no contexto da Atenção Primária à Saúde (APS), reorganizar os fluxos de distribuição com vistas a garantir às pessoas o acesso aos serviços e tratamentos, a partir da manutenção do fornecimento de insumos a pacientes com doenças crônicas (hipertensão e diabetes) e pessoas dependentes de medicamentos de uso ambulatorial. A garantia do acesso ao medicamento pelo Sistema Único de Saúde (SUS), para muitos pacientes, é a única possibilidade de tratamento, de modo que é imprescindivel que os tratamentos, mesmo considerando a orientação de isolamento social, com reclusão aos domicílios, sejam realizados durante a pandemia.

No que concerne às novas necessidades oriundas da pandemia de Covid-19, a Coordenadoria da Assistência Farmacêutica, alinhada às Coordenadorias Jurídica e Administrativo-financeira da Secretaria da Saúde de Sobral, deflagraram processos para a efetivação de compras de medicamentos e insumos para a garantia de retaguarda aos hospitais de campanha. A aquisição é a etapa do ciclo da assistência farmacêutica que se faz mediante exigências legais e logísticas a serem cumpridas pelo setor público de saúde. Embora seja necessário um processo de licitação, o que gera eficiência, legalidade e moralidade no proceder da compra de insumos para a promoção, proteção e recuperação da saúde, tanto individual como coletiva ${ }^{4}$, nos casos de emergência ou calamidade pública, a exemplo da que o país se encontra frente à pandemia, a licitação é dispensável, conforme estabelecido no Decreto Legislativo n. ${ }^{0}$ 6, de 2020, promulgado pelo Congresso Nacional ${ }^{5}$.

Considerando a experiência da atuação da força de trabalho da Coordenadoria da Assistência Farmacêutica e a necessidade de reorganização das ações desta coordenadoria em face da pandemia de Covid-19, objetiva-se, por meio deste estudo, relatar as contribuições da assistência farmacêutica para 0 enfrentamento do novo coronavírus, no município de Sobral, Ceará, com ações voltadas para a promoção, proteção e recuperação da saúde, garantindo os princípios da universalidade, integralidade e equidade.

\section{METODOLOGIA}

Trata-se de um relato de experiência desenvolvido

\author{
"Sobral tem cobertura \\ de $100 \%$ na Atenção \\ Primária à Saúde (APS) \\ por meio da Estratégia \\ Saúde da Família (ESF)"
}

a partir da experiência da Coordenadoria da Assistência Farmacêutica, no curso da Covid-19, no município de Sobral, Ceará. Refere-se ao período de março a outubro de 2020.

$0 s$ relatos de experiências representam uma possibilidade de criação de uma narrativa científica que se reveste de importância por englobar processos e produções subjetivas aplicadas. Estes escritos perfomatizam, por meio da linguagem, a experiência não enquanto centralidade estável, mas na condição de ponto de abertura e análise crítica. Situam, portanto, o saber resultante de um processo que considera as relações e aprendizagens de uma pessoa ou grupo ${ }^{6}$.

Sobral tem cobertura de $100 \%$ na Atenção Primária à Saúde (APS) por meio da Estratégia Saúde da Família (ESF). A retaguarda da Coordenadoria da Assistência Farmacêutica é uma constante nesse cenário. No curso da pandemia, outras atividades foram incorporadas a essa coordenação, a exemplo dos hospitais de campanha implantados.

A descrição dos processos delineados e implementados é realizada por sujeitos que os vivenciaram, a partir de um olhar crítico por um retrovisor que objetiva registrar experiências como fonte de socialização e que, ao mesmo tempo, reflete sobre uma vivência com finalidade de agregar lições aprendidas à prática profissional.

\section{RESULTADOS E DISCUSSÃO}

A assistência farmacêutica passou por modificações significativas diante das novas necessidades dos serviços de saúde e dos usuários, no decorrer da pandemia de Covid-19. Com o intuito de maximizar a eficiência e, alinhada às diretrizes municipais do Plano Municipal de Contingência de Sobral, Ceará, a Coordenadoria de Assistência Farmacêutica reestruturou todo o aporte de insumos e medicamentos, além de redefinir os fluxos. Esses processos serão apresentados e analisados em dois tópicos: Assistência Farmacêutica e Atenção Primária 
em tempos de Covid-19: colaboração para a garantia do cuidado e Assistência Farmacêutica na retaguarda dos hospitais de campanha.

\section{Assistência Farmacêutica e Atenção Primária em tempos de Covid-19: colaboração para a garantia do cuidado}

A Atenção Primária à Saúde, reafirmando a sua função estratégica, desempenhou importante papel no enfrentamento da pandemia de Covid-19, desde o acompanhamento longitudinal ao desenvolvimento de ações de promoção da saúde e prevenção de agravos. Com isso, a definição do isolamento e distanciamento social para retardar a expansão da pandemia e possibilitar a adequação dos serviços de saúde ao rápido aumento da demanda por assistência ${ }^{7}$ resultou na diminuição da circulação de pessoas, implicando na redução do acesso dessas às Unidades Básicas de Saúde $(U B S)^{8}$. Diante desse cenário, algumas medidas emergenciais precisaram ser tomadas para garantir a segurança daquelas pessoas que necessitavam dos serviços dos equipamentos de saúde, assim como daqueles que estavam reclusos aos seus domicílios requerendo cuidados de profissionais de saúde e/ou medicamentos e insumos.

Nesse contexto, destacam-se as pessoas com doenças e/ou agravos crônicos à saúde, tais como: hipertensão, diabetes e transtornos mentais, as quais requeriam da APS a continuidade do cuidado e atenção especial para a prevenção de contágio pelo SARSCov-2 (oriundo do inglês: severe acute respiratory syndrome coronavirus 2), uma vez que se configuravam grupo de risco para a Covid-19. Como resposta a essa necessidade, a assistência farmacêutica do município reorganizou o fluxo de atenção a esses pacientes, no que compete ao fornecimento das medicações de uso regular, a saber: ampliação da validade dos receituários emitidos, estratégia remota de solicitação de renovação de receituários do componente especializado e organização do fluxo de dispensação de medicamentos.

Para os receituários emitidos no curso da pandemia foi instituído que a validade dos mesmos fosse ampliada de 30 para 180 dias, para que os pacientes não necessitassem de forma rotineira ir à UBS. Com essa medida, garantia-se que não ocorresse descontinuidade do uso da medicação por falta desta e que os usuários dessa terapêutica se mantivessem mais seguros em casa, cumprindo o isolamento social. Essa ação foi ancorada nas Leis n. ${ }^{0} 13.979$, de

\section{"Na impossibilidade de locomoção do usuário, foi utilizado o serviço de entrega das medicações, também pelo Agente Comunitário de Saúde $(A C S)^{\prime \prime}$}

6 de fevereiro de 2020 e n. ${ }^{\circ} 14.028$, de 27 de julho de 2020, que versam, respectivamente, sobre as medidas emergenciais a serem adotadas no período da pandemia, incluindo as orientações relativas à prescrição e validade de receituários emitidos por médicos ou odontólogos ${ }^{9-10}$.

No que se refere à dispensação de medicamentos de monitoramento e controle da Farmácia de Medicamentos Especiais, que dispensa as medicações do Componente Especializado da Atenção Farmacêutica, adotou-se imediatamente a declaração da emergência sanitária e a orientação de que a renovação de receituário ocorreria via telefone ou e-mail. Para responder prontamente, foi designado um profissional para monitorar, processar e encaminhar as solicitações que chegavam por meio desses canais de comunicação.

Posteriormente, a equipe de tecnologia da informação da Secretaria da Saúde criou um sistema de agendamento on-line, em que a população agendava o dia e a hora para receber a medicação, respeitando as medidas sanitárias e evitando aglomerações. $\mathrm{Na}$ impossibilidade de locomoção do usuário, foi utilizado o serviço de entrega das medicações, também pelo Agente Comunitário de Saúde (ACS), que fora orientado quanto às medidas protetivas para a redução dos riscos de contaminação nas visitas domiciliares. A adequação dos fluxos de trabalho nas atividades de rotina da APS, principalmente no que diz respeito à dispensação de medicações, foi reconhecida como uma estratégia necessária ${ }^{11}$.

Esse conjunto de estratégias visou garantir a assistência farmacêutica à população de maneira a assegurar a continuidade do cuidado, protegendo-a de riscos adicionais de exposição, uma vez que são exatamente esses grupos populacionais que apresentam comorbidades crônicas que requerem especial atenção e que são mais susceptíveis ao adoecimento e às complicações da Covid-19. De acordo com estudo publicado pelo Centro Chinês de 
Controle e Prevenção de Doenças, as comorbidades mais frequentes nos pacientes que evoluíram ao óbito foram hipertensão arterial, diabetes mellitus, doenças cardiovasculares e idade acima de $70 \operatorname{anos}^{12}$. Nesse cenário, a assistência farmacêutica se insere na conformação de uma ação multiprofissional no adequado manejo das condições clínicas desses usuários, com compromisso para a continuidade do tratamento ${ }^{13}$.

Até o momento, não há alternativa terapêutica farmacológica eficaz para o tratamento da Covid $-19^{8}$. Como consequência, mesmo sem fortes evidências científicas, muitos medicamentos têm sido utilizados empiricamente na tentativa de diminuir os sintomas e a letalidade da doença. 0 uso de Hidroxicloroquina e a Cloroquina gerou polarização quanto à sua indicação, por ausência de estudos robustos que demonstrem eficácia dessa medicação na redução da mortalidade ou melhora dos desfechos clínicos em pacientes acometidos pela Covid-19. Outrossim, estes integraram as orientações de utilização, em casos específicos, na terapêutica para Covid-19, apresentada pela Secretaria de Saúde do Estado do Ceará ${ }^{14}$.

A gestão municipal da saúde e assistência farmacêutica optaram por aderir às recomendações de tratamento farmacológico ambulatorial para pacientes adultos com quadros suspeitos ou confirmados de Covid-19, indo de acordo com a nota técnica da Secretaria de Saúde do Estado do Ceará. A partir de criteriosa avaliação médica, presencial ou remota, prescreve-se o esquema terapêutico de Prednisona e Azitromicina. Junto com a prescrição, o médico e o paciente deverão assinar um Termo de Consentimento Informado sobre o tratamento ${ }^{15}$. Vale ressaltar que esses medicamentos fazem parte do elenco da relação básica de medicamentos do município de Sobral, portanto a assistência farmacêutica teria como garantir o abastecimento da medicação de acordo com os critérios estabelecidos para o uso racional.

Reconhecendo que a maioria dos casos de Covid-19 apresenta sintomatologia leve e que a APS é a principal porta de entrada à Rede de Assistência à Saúde (RAS), é importante reconhecer também o seu papel estratégico frente a essa pandemia, assim como é necessária a parceria com a assistência farmacêutica para a garantia de efetiva assistência orientada pelo uso racional de medicamentos, uma vez que a práxis iatrogênica sem fortes evidências pode gerar riscos à saúde e consequências irreversiveis ${ }^{16}$.

\section{"Até o momento, não há alternativa terapêtica farmacológica eficaz para o tratamento da Covid-19"}

É válido salientar que a garantia de insumos, particularmente aqueles dirigidos à proteção dos trabalhadores da saúde, foi outro desafio vivido pela Coordenadoria de Assistência Farmacêutica, que também tem a incumbência de provê-los. Nesse contexto, assumiram importância as estratégias de gestão de estoque para garantir a segurança e qualidade da assistência para pacientes e trabalhadores da saúde.

Desde os primeiros casos de Covid-19, alguns Equipamentos de Proteção Individual tornaramse cada vez mais raros. Diversos países, inclusive - Brasil, têm registrado falhas na proteção de trabalhadores da saúde por conta da escassez desses equipamentos ou devido a equívocos na paramentação ou desparamentação ${ }^{17}$. 0 uso correto e racional dos EPIs pelos profissionais de saúde é fundamental para evitar a contaminação e disseminação do SARSCoV-2. A Coordenadoria da Assistência Farmacêutica, reconhecendo essa necessidade, interveio no sentido de adquirir e disponibilizar suficientemente esses equipamentos para todos os trabalhadores. Para além do acesso aos EPIs, todos os profissionais passaram por capacitações promovidas pela Escola de Saúde Pública Visconde Saboia (ESP-VS) quanto ao uso adequado, baseado nas recomendações da Organização Mundial da Saúde (OMS), com definição de quais os tipos de EPIs são indicados e adequados conforme cada situação de trabalho; evitando, assim, o desperdício e aumentando o custo-efetividade. As recomendações para o uso racional de EPI buscaram orientar as pessoas envolvidas na distribuição e no gerenciamento desses produtos.

\section{Assistência Farmacêutica na retaguarda dos hospitais de campanha}

Considerando a necessidade de organizar a atenção hospitalar, sob gestão pública municipal para o enfrentamento ao estado de emergência em saúde pública, com a indispensável garantia do 
atendimento à saúde da população de forma digna, séria, responsável, profissional e com o devido respeito, foram implantados dois hospitais de apoio às ações contra a Covid-19. 0 Decreto n. ${ }^{0} 2.369$, de 13 de março de 2020, declara estado de perigo público iminente na rede hospitalar do município de Sobral e decreta intervenção municipal por modalidade de requisição do prédio e de todas as instalações físicas do Hospital Doutor Estevam Ponte, englobando tudo o que seja necessário para o seu regular funcionamento, em benefício do atendimento dos que dele necessitam ${ }^{18}$. 0 Decreto n..$^{0} 2.377$, de 20 de março de 2020, declara a intervenção municipal por modalidade de requisição do prédio e de todas as instalações físicas da Clínica Doutor Francisco Alves, englobando tudo o que seja necessário para o seu regular e efetivo funcionamento, em benefício dos que dele necessitam ${ }^{19}$. Esses hospitais estavam inativados ou subutilizados e passaram a compor a rede de atenção à saúde.

A administração do hospital de campanha foi diferenciada das experiências ocorridas em muitos cenários do território nacional. 0 município de Sobral assumiu a responsabilidade de gerir e administrar os hospitais de campanha, indo na contramão do que estava ocorrendo em muitos estados e municípios no país, com os gestores firmando parcerias com o setor privado ou serviços filantrópicos. Essa cooperação ocorreu, muitas vezes, por administração indireta e terceirizada da gestão de serviços de saúde pública e grandes hospitais privados, algo que já ocorria anteriormente em todo o país ${ }^{20}$.

Essa decisão trouxe grandes desafios para a assistência farmacêutica, pois embora o município fosse referência na média e alta complexidade em saúde, não possuía hospital municipal. A garantia de um planejamento e aquisição de medicamentos e insumos fez com que a assistência farmacêutica lidasse com o desafio de otimizar os recursos financeiros, com a retração da oferta e a importância de garantia de preços não-abusivos numa situação de emergência, tudo isso dentro do arcabouço e dos requisitos da legislação brasileira de compra e aquisição de medicamentos ${ }^{21}$.

Os critérios de eficácia e segurança foram a base para a seleção de itens que compuseram - subconjunto de medicamentos dos hospitais. Destarte, esta etapa é uma administração técnica, política e administrativa do processo. A seleção de medicamentos é de suma importância sendo o eixo norteador para o planejamento e orientação das

\section{"Sobral assumiu a responsabilidade de gerir e administrar os hospitais de campanha, indo na contramão do que estava ocorrendo em muitos estados e municípios"}

etapas seguintes desse ciclo e da ação clínica ${ }^{22}$.

Para suprir as necessidades de medicamentos e material médico, a padronização das medicações utilizadas foi fundamental. Essa estratégia teve como objetivo nortear a gestão da assistência farmacêutica, na medida em que definia necessidades, prioridades e direcionava a aplicação de recursos financeiros. A padronização foi ancorada por evidências científicas e sua elaboração seguiu instrumentos normativos e protocolos clínicos com a avaliação do uso da medicação e a análise de custo-efetividade. Frente à situação de pandemia e o crescente número de óbitos gerados pela Covid-19, esse processo precisou de agilidade e cautela visto que o tratamento requerido era de urgência e deveria gerar resultados promissores com a melhora do quadro de evolução dos pacientes a serem tratados.

0 acesso às tecnologias de saúde se constitui em uma condição necessária para a efetividade e segurança do tratamento, especialmente em pacientes críticos. As dificuldades envolvendo os abastecimentos é um problema que atinge os serviços de saúde, provocando a ruptura de estoque de medicamentos e produtos. As maiores dificuldades de abastecimento envolvem medicamentos para a sedação, bloqueio neuromuscular e analgesia. Considerando a recomendação da Sociedade Brasileira de Anestesiologia, preocupados pela escassez de fármacos essenciais para a anestesia e analgesia, sedação e relaxamento muscular, neste momento de pandemia ${ }^{23}$, a assistência farmacêutica recomenda o uso racional desses medicamentos, evitando o desabastecimento e o sofrimento do paciente.

\section{CONCLUSÃO}

A assistência farmacêutica enfrentou diversos desafios organizativos no contexto da pandemia por Covid-19, como a garantia do cuidado longitudinal e integral às pessoas com condições crônicas, abastecimento de serviços da APS e hospitais de 
campanha, além da aquisição de medicamentos dentro dos parâmetros legais.

Este relato evidencia a contribuição da assistência farmacêutica no enfrentamento à Covid-19, em Sobral, Ceará, a partir da garantia de logística para a aquisição de medicamentos, estando atenta aos princípios da Administração Pública, acompanhamento do fluxo ao longo da cadeia de fornecimento, garantindo uma entrega rápida, segura e praticabilidade de controle e informação, evitando o desabastecimento de medicamentos e insumos das unidades, sempre buscando estratégias para facilitar o acesso aos medicamentos e continuidade do cuidado.

Espera-se que este estudo possa ser útil para o desenvolvimento de novas pesquisas relacionadas à assistência farmacêutica e na preparação do setor de saúde para emergências e desastres em saúde pública.

\section{CONTRIBUIÇÃO DOS AUTORES}

Estevam Ferreira da Ponte Neto contribuiu com redação, análise e revisão crítica do manuscrito. Tereza Doralúcia Rodrigues Ponte contribuiu com redação, análise e revisão crítica do manuscrito.

Delano de Sousa Aragão contribuiu com redação, análise e revisão crítica do manuscrito. Pedro Henrique Martins contribuiu com redação, análise e revisão crítica do manuscrito.

\section{REFERÊNCIAS}

1. Brasil. Resolução n. ${ }^{\circ} 338 /$ CNS/MS, de 6 de maio de 2004. Aprova a Política Nacional de Assistência Farmacêutica. Diário Oficial da União [document on the internet], Brasília (2004 May 20 [cited 2020 Nov 20]). Sec 1, p. 52. Available from: http:// bvsms.saude.gov.br/bvs/saudelegis/cns/2004/ res0338 $06 \quad 05 \quad 2004 . h t m l$

2. Estados Unidos da América. Comprehensive Hospital Preparedness check-list for Coronavirus Disease 2019 (COVID-19). Centro de Controle e Prevenção de Doenças [document on the internet]; 2020. [cited 2020 Nov 23]. Available from: https:// www.cdc.gov/coronavirus/2019-ncov/hcp/hcphospital-checklist.html

3. World Health Organization. Meeting Report: Technical Definitions of Shortages and Stockouts of Medicines and Vaccines. Geneva: World Health Organization; 2016.
4. Brasil. Lei n. ${ }^{\circ} 8.666$, de 21 de junho de 1993. Regulamenta o art. 37, inciso XXI, da Constituição Federal, institui normas para licitações e contratos da Administração Pública e dá outras providências. Diário Oficial da União [document on the internet], Brasília (1993 June 21 [cited 2020 Nov 22]). Sec 1. Available from: http://www.planalto.gov.br/ ccivil 03/leis/l8666cons.htm

5. Brasil. Decreto Legislativo n. ${ }^{0}$ 6, de 2020. Reconhece, para os fins do art. 65 da Lei Complementar n. ${ }^{\circ} 101$, de 4 de maio de 2000, a ocorrência do estado de calamidade pública. Diário Oficial da União [document on the internet], Brasilia (2020 Mar 18 [cited 2020 Nov 20]). Sec 1. Available from: https://www.planalto.gov.br/ccivil 03/portaria/ dlg6-2020.htm

6. Daltro MR, Faria AA. Relato de experiência: Uma narrativa científica na pós-modernidade. Psicologia clínica e psicanálise [serial on the internet] 2019 [cited dez 01]; 19(1). Available from: https:// www.e-publicacoes.uerj.br/index.php/revispsi/ article/view/43015/29664

7. Prem K, Liu Y, Russell TW, Kucharski AJ, EgGo RM, Davies $N$, et al. The effect of control strategies to reduce social mixing on outcomes of the COVID-19 epidemic in Wuhan, China: a modelling study. Lancet Public Health [serial on the internet] 2020 [cited 2020 Nov 24]; 5:e261-e70. Available from: https://www.thelancet.com/ action/showPdf?pii=S2468-2667\%2820\%293 $\underline{0073-6}$

8. Soeiro RE, Bedrikow R, Ramalho BDS, Niederauer AJS, Souza CV, Previato CS, et al. Atenção Primária à Saúde e a pandemia de COVID-19: reflexão para a prática. InterAmerican Journal of Medicine and Health [serial on the internet] 2020 [cited 2020 Nov 24]; 3. Available from: https://www.iajmh.com/ iajmh/article/view/83

9. Brasil. Lei n. ${ }^{0} 13.979$, de 06 de fevereiro de 2020. Dispõe sobre as medidas de enfrentamento da emergência de saúde pública de importância internacional decorrente do coronavírus responsável pelo surto de 2019. Diário Oficial da União [document on the internet], Brasilia (2020 Fev 6 [cited 2020 Nov 20]). Sec 1. Available from: https://www.in.gov.br/en/web/dou/-/lei-n13.979-de-6-de-fevereiro-de-2020-242078735

10. Brasil. Lei n. ${ }^{0} 14.028$, de 27 de julho de 2020 . Altera a Lei n ${ }^{0} 13.979$, de 06 de fevereiro de 2020, para garantir o receituário médico ou odontológico de medicamentos sujeitos a prescrição e de uso contínuo tenha validade pelo menos enquanto perdurarem as medidas de isolamento para contenção do surto da Covid-19, na forma que específica. Diário Oficial da União [document on the internet], Brasília (2020 July 27 [cited 2020 Nov 20]). Sec 1. Available 
from: https://www.in.gov.br/en/web/dou/-/lei-n14.028-de-27-de-julho-de-2020-268918657

11. Gurtler CA, Corrêa BC, Gurtler MRB, Menezes MSB, Salvetti MCP. Gestão de estoques no enfrentamento à pandemia de COVID-19, 2020. Revista Qualidade HC [serial on the internet]. 2020 [cited 20200 ct 22]; 71-81. Available from: https://www.hcrp.usp.br/revistaqua lidade/uploads/Artigos/250/250.pdf

12. Zhonghua L, Xing BX, Za Z. The Novel Coronavirus Pneumonia Emergency Response Epidemiology Team. The epidemiological characteristics of an outbreak of 2019 novel coronavirus diseases (COVID-19) in China. Centers for Disease Control and Prevention [serial on the internet]. 2020 [cited 2020 0ct 22]; 41(2):145-51. Available from: https://slma.lk/ wpcontent/uploads/2020/02/TheEpidemiologicalCh aracteristicsofan 0utbreakof 2019 NovelCoronavirusDi seases28COVID-1929E28094China2C20201.pdf

13. Alves MTG. Reflexões sobre o papel da Atenção Primária à Saúde na pandemia de COVID-19. Revista Brasileira de Medicina de Família e Comunidade [serial on the internet]. 2020 [cited 2020 Nov 29]; 15(42):2496-96. Available from: https://rbmfc.org. $\underline{\mathrm{br} / \mathrm{rbmfc} / \mathrm{article} / \mathrm{view} / 2496}$

14. Ceará. Nota Técnica sobre o uso das medicações Hidroxicloroquina e Cloroquina como drogas experimentais para o tratamento de Covid-19 [document on the internet]. Ceará: Secretaria de Saúde do Estado do Ceará; 2020 [cited 2020 Nov 29]. Available from: https://www.saude.ce.gov.br/wpcontent/uploads/sites/9/2020/02/nota-tecnica usos-da-hidroxocloroquina-e-cloroquina V2.pdf

15. Ceará. Recomendações de tratamento farmacológico ambulatorial para pacientes adultos com quadro suspeito ou confirmado de Covid-19 [document on the internet]. Ceará: Secretaria de Saúde do Estado do Ceará; 2020 [cited 2020 Nov 30]. Available from: https://www.s a ude.ce.gov.br/wpcontent/uploads/ sites $/ 9 / 2020 / 02 /$ nota tecnica de tratamento farmacologico ambulatorial 0405 2020.pdf

16. Tritany RF, Tritany EF. Uso Racional de Medicamentos para COVID-19 na Atenção Primária à Saúde. Saúde em Redes [serial on the internet]. 2020 [cited 2020 Nov 29]; 6(2). Available from: http:// revista.redeunida.org.br/ojs/index.php/rede-unida/ article/view/3205

17. Soares SSS, Souza NVD, Silva KG, César MP, Souto JDSS, Leite JCRAP, et al. Pandemia de Covid-19 e o uso racional de equipamentos de proteção individual [Covid-19 pandemia e uso racional de equipamentos de proteção individual] [Pandemia de Covid-19 e uso racional de equipamentos de proteção pessoal]. Revista enfermagem UERJ [serial on the internet]. 2020 [cited 2020 Nov 13]; 28:50360. Available from: https://www.epublicacoes.uerj.br/index.php/enfermagemuerj /article/download/50360/34044

18. Sobral. Decreto n. ${ }^{0} 2.369$, de 13 de março de 2020 - Declara estado de perigo público iminente na rede hospitalar do município de Sobral, e decreta intervenção municipal por modalidade de requisição do prédio e de todas as instalações físicas do Hospital Doutor Estevam Ponte. Diário Oficial do Municipio [document on the internet], Sobral, ano IV, no 752 (2020 Mar 13 [cited 2020 Nov 22]). Available from: http://www.sobral. ce.gov.br/diario/public/files/diario/00ebe56060c94 df734b04637524ca2eb.pdf

19. Sobral. Decreto n. ${ }^{0} 2.377$, de 20 de março de 2020 - Declara a intervenção municipal por modalidade de requisição do prédio e de todas as instalações físicas da Clínica Doutor Francisco Alves, englobando tudo que seja necessário para o seu regular e efetivo funcionamento, em benefício dos que dele necessitam. Diário Oficial do Município [document on the internet], Sobral, ano IV, ${ }^{\circ} 757$ (2020 Mar 20 [cited 2020 Nov 22]). Available from: http://www.sobral. ce.gov.br/diario/public/files/diario/09e9f46bffe $4 \mathrm{fd}$ e091b9d116a5f7e999.pdf

20.Sodré F. Epidemia de Covid-19: questões críticas para a gestão da saúde pública no Brasil. Trabalho, Educação e Saúde [serial on the internet]. 2020 [cited 2020 Nov 14]; 18(3):e00302134. Available from: https://www.scielo.br/scielo. php?pid=S1981-77462020000300401\&script $=$ sci arttext

21. Chaves LA, 0sorio-de-Castro CGS, Caetano MC, Silva RAL, Luiza VL, et al. Nota técnica. Desabastecimento, uma questão de saúde pública global: sobram problemas, faltam medicamentos. Fiocruz [document on the internet]. 2020 [cited 2020 Nov 14]. Available from: https://www.arca.fiocruz. br/bitstream/icict/42974/2/Desabastecimento medicamentos.pdf

22. Karnikowski MG0, Galato D, Meiners MMM A, Silva EV, Gerlack LF, Bós Ângelo JG, et al. Characterization of the selection of medicines for the Brazilian primary health care. Revista de saúde pública [serial on the internet]. 2017 [cited 2021 Nov 15]; 51(suppl.2):9s. Available from: https://www.revistas.usp.br/rsp/ article/view/139742

23. Diego LAS, Detoni PB, Azevedo MP, Carraretto AR, Albuquerque MAC, Tardelli MA, et al., Recomendação da Sociedade Brasileira de Anestesiologia (SBA) para o Uso Racional de Fármacos para Anestesia e Sedação durante a Covid-19. Sociedade Brasileira de Anestesiologia [document on the internet]; 2020 [cited 2021 Nov 15]. Available from: https://www.crmpr. org.br/uploadAddress/recomendacao-da-sba-parao-uso-racional-de-farmacos-para-anestesia-esedacao-durante-a-covid-19[4471].pdf

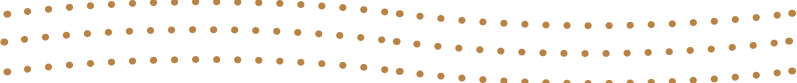

